\title{
Local Strain Measurement of Kevlar Strand with Fiber Optic Bragg Grating
}

\author{
Curtis E. Banks ${ }^{1}$, Joseph Grant ${ }^{2}$, Sam Russell ${ }^{3}$ and Shawn Arnett ${ }^{4}$ \\ ${ }^{1}$ Integrated Structure Health Monitoring and Sensors Branch \\ EV43 MSFC/NASA, Huntsville, Al 35812 \\ ${ }^{2}$ Science \& Technology Division \\ MSFC/NASA, Huntsville, Al 35812 \\ ${ }^{3}$ Nondestructive Evaluation Branch \\ EM20 MSFC/NASA, Huntsville, Al 35812 \\ ${ }^{4}$ TRI/Austin, Inc. \\ 9063 Bee Caves Rd., Austin, Texas 78733
}

\begin{abstract}
1987 DuPont manufactured 4560 denier Kevlar/Epoxy Strands were instrumented with nine and three sensors each. Stress tests were performed at 30,45,60,70 and $80 \%$ of ultimate strength with dwell times of 10,000 seconds. FBG showed uneven stress levels which is contrary to conventional observation.
\end{abstract}

\section{Introduction}

Stress rupture in Composite Overwrapped Pressure Vessel (COPV) is a major concern to NASA because studies reveled that the probability of stress rupture leading to catastrophic failure were greater than previously though ${ }^{1}$. All COPV structure degrades over time make stress rupture more likely ${ }^{2}$. Kevlar COPVs was used extensively on the space shuttle because of their weight saving advantages ${ }^{3}$. The energy of a COPV structure is held by the overwrap, where as the liner is mainly used to retain the commodity. DuPont 19874660 denier Kevlar (strand or tow) was used to wrap these vessels.

To investigate stress rupture a team of nondestructive evaluation (NDE) experts were assembled to employ the latest NDE techniques to predict stress rupture. As a means of developing Fiber optic Bragg Gratings as a viable NDE and Integrated Vehicle Health Monitoring (IVHM) technique, FBGs were applied to the Kevlar during tensile creep test to measure local stress alone the tow of the fiber. It is know that localized high stress field is the likeliest place for stress rupture ${ }^{1}$.

The Kevlar strand is comprised of several smaller bundles making to 0.1 inch the strand. The FBG sensor is small compared to the tow making it possible to measure the uneven strain fields under stress.

All tests were carried out at Texas Research Institute Inc., Austin, Texas.

\section{Experimental Testing and Results}

Three ten inch 4650 denier Kevlar epoxy strands were instrumented with FBGs strain sensors and prepared for tensile creep testing (figure 1). Grip attachment were placed on 
each end of the strands and affixed to a cardboard backing for handling. One strand was instrumented with nine sensors. This strand had a total of three FBG cables with three sensors each. On the other hand, the two remaining strands had three sensors each. Their spacing was two and three-quarters inch while the first strand spacing was a little over an inch. At each sensor location the sensor was pulled taunt and glued to the strands with Vishay M-bond AE-10 epoxy.

These samples were individually placed inside the instrum for creep testing. Under stress, a Micron Optic sm125 FBG interrogator was used to track the peak shift of the sensors. The predetermined strain loads were $30,40,60,70$, and $80 \%$ of the ultimate load strength of the strand $(212 \mathrm{lbs})$. The ambient temperature was kept at 34 degrees. At each increment the dwell time was 10,000 seconds. This allowed the strands to undergo appreciable creep levels.

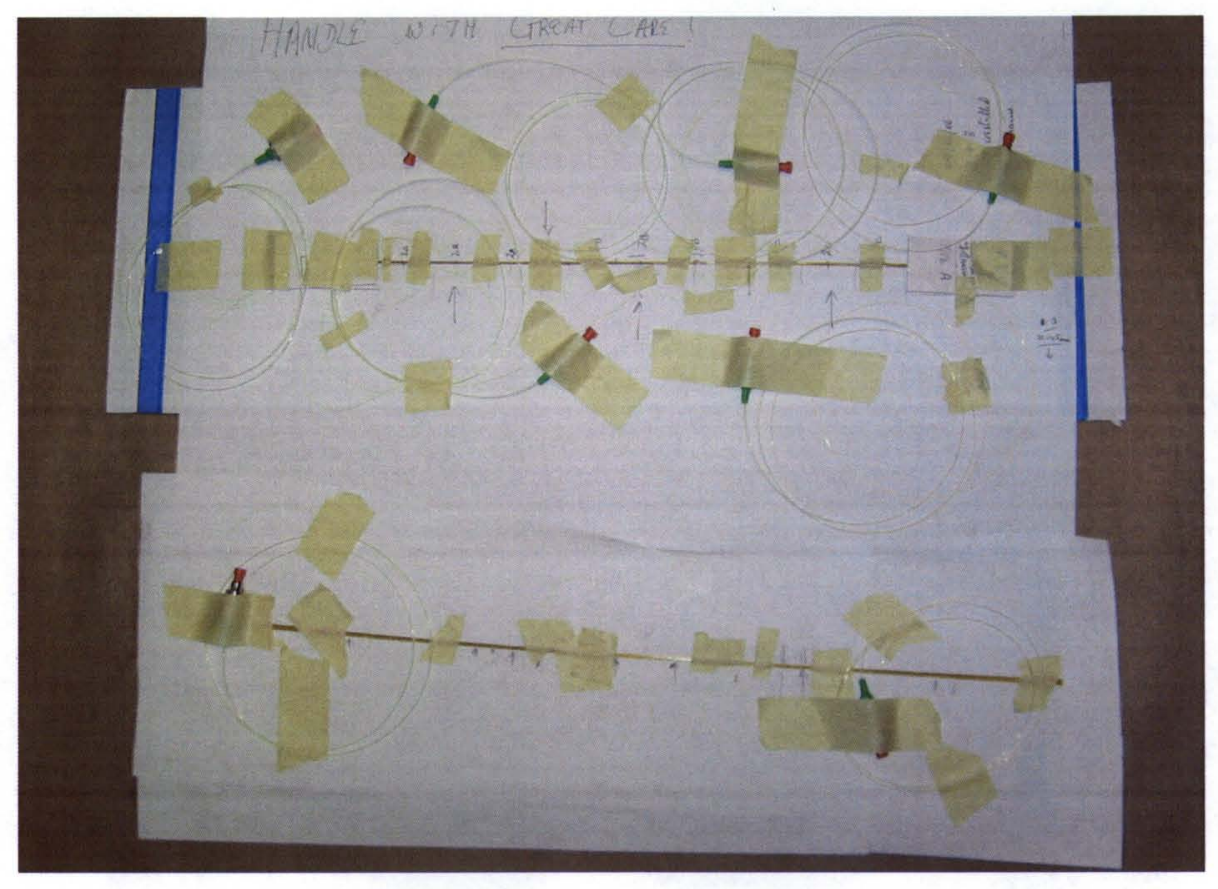

Figure 1 - Kevlar/Epoxy Strands with Bonded FBGs

Of most significance in this test was the functioning of the FBGs and the resultant file creation by the sm 125 software. As stress was applied to the strand, peak splitting occurred at various wavelengths. When the program recognizes additional peaks, their wavelengths and power levels $(\mathrm{dB})$ were appended to the text file. This would happen especially when the stress levels were changed. Should a peak stop being recognized, no appended data was written. This created a significant problem in Excel sorting the data to align the columns properly. A feature of the text file was used to sort the data. The first column of the file was elapsed time in seconds. The next four show the number of peaks being identified for each active channel. Therefore, by using logic formulas to copy data to new columns (based on the exact number of peaks being identified) allowed all of those with 3, 4, 5, 6 or 7 to be aligned separately. However, where a subset had blanks, Excel charted them as zeros, hence the row of symbols across the 0 axis. Another problem became apparent that is shown in Figure 2. Although the sorting based on 
number of peaks (columns) helped, they were not uniformly consistent regarding the correct wavelengths. This is visible in the subset of data later in the test around 12,000 microstrain. The microstrains were determined by using a conversion factor of 1.300 picometers per 1,000 microstrain.

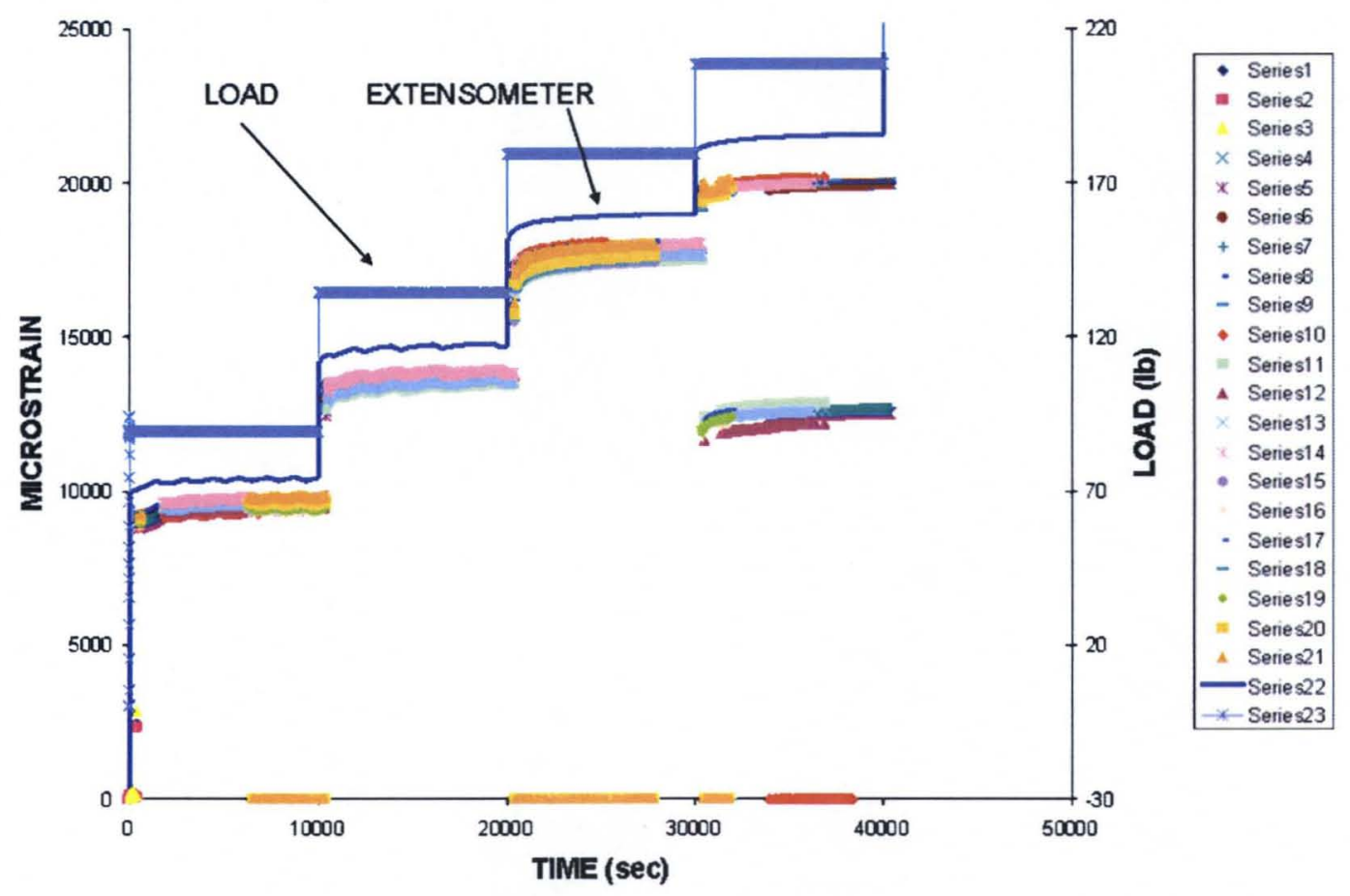

Figure 2 - Extensometer and FBG Microstrain and Load Data for First Strand Test (the FBG data is plotted in symbols to prevent vertical lines from connecting entries seen as zeroes)

Some peaks appeared and disappeared throughout the test that varied in wavelength. This data could have been corrected by changing the normalizing wavelength to its correct assignment, but it is important to illustrate this aspect of the data collection and operation of the test. Figure 3 shows the peak wavelengths detected throughout the test. They were "self-sorting" in a manner of speaking in that all were recorded regardless of which series they were in. Figure 4 shows a portion of the test in higher resolution to illustrate the range of strains that multiple peaks represent. This spread of around 800 microstrain is not conducive to accurate strain measurement. The curves shapes do, however, match the extensometer very well, but they indicate that the original gage length does not apply to them all.

Some of these problems could be minimized if the operator is present and able to modify the peak detection parameters in the operating software. This is not practical for long term tests, and it is problematic in that multiple or attenuated peaks are difficult to sort properly on a single channel's parameter settings.

It is believed that the cause of the peak splitting has to do with the introduction of either twisting or bi-axial stresses. Our conclusion is that strands are not easily tested with FBGs due to surface changes under stress that apparently impart compressive or torsional stresses on the fiber. As will be discussed later, applying FBGs to a COPV can also be 
affected by surface changes in the composite under stress and require special bonding techniques to minimize the effects.

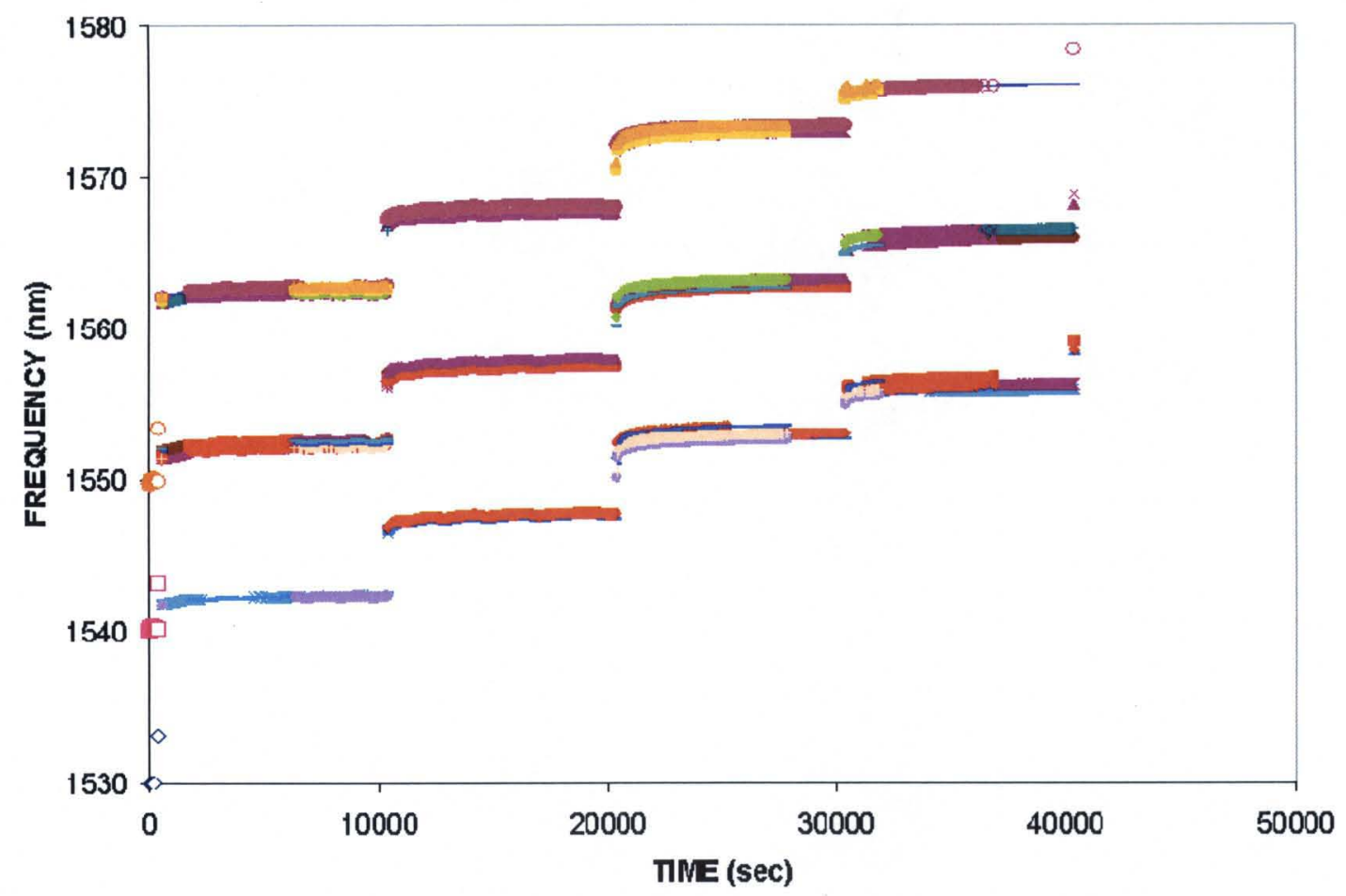

Figure 3 - Peak Wavelengths Increasing with Increasing Stress Levels

The power level data is shown in Figure 5 below. Compared to peak power level attenuation on "embedded" FBGs on COPV liners, these are fairly modest losses. The strand with nine FBGs was then tested; however, installation of an extensometer was not possible so only crosshead displacement data was collected in addition to the sensors' data. The crosshead displacement does not provide a very useful method of determining a tensile specimen's true strain and due to various grip, specimen and load frame compliances will invariably record higher computed strains than would be measured locally on the specimen with an extensometer, strain gage or other strain sensor (i.e., FBG). 


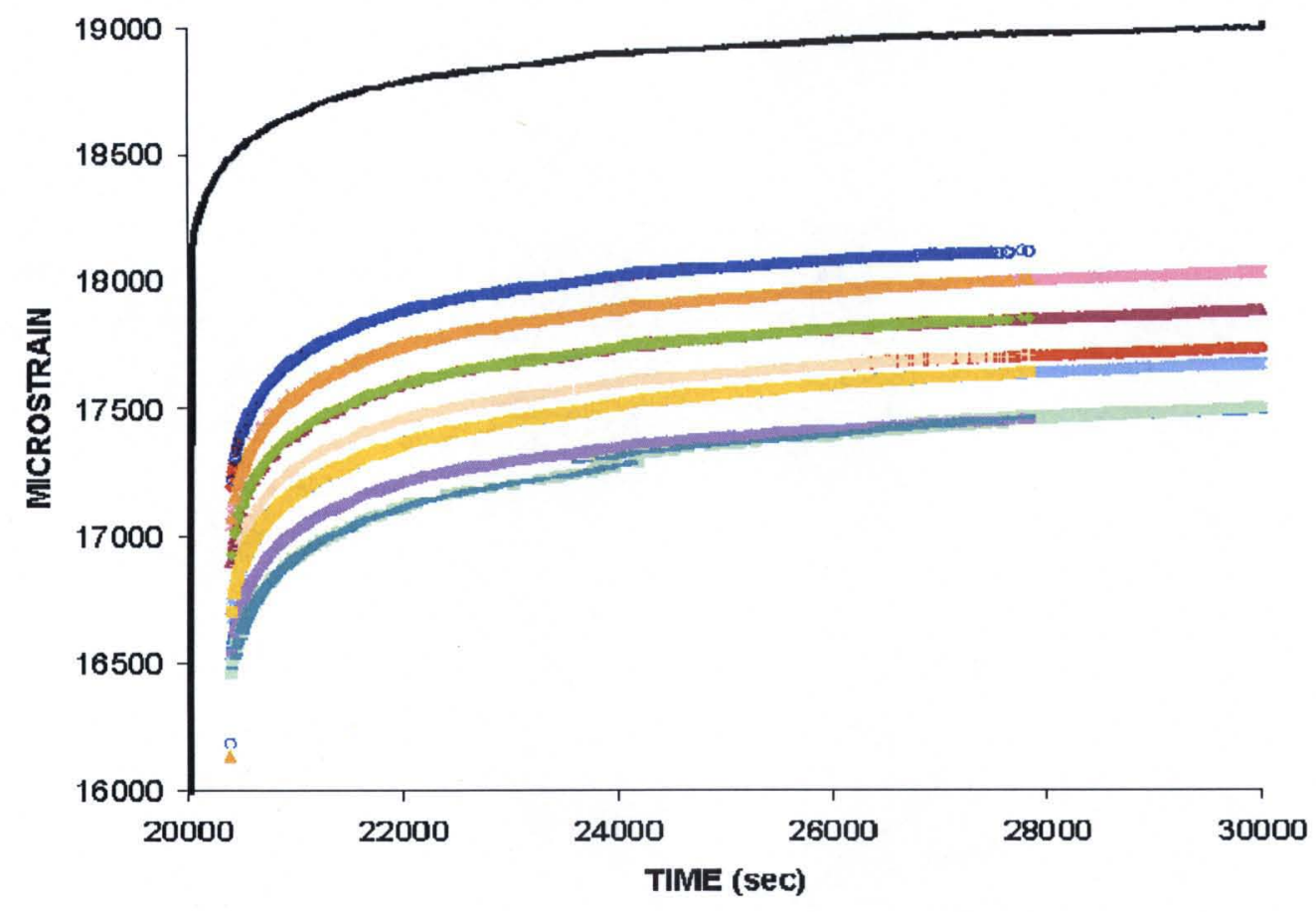

Figure 4 - Close-up View of Part of the Test to Illustrate the Spread in Microstrain (the highest curve is the extensometer output)

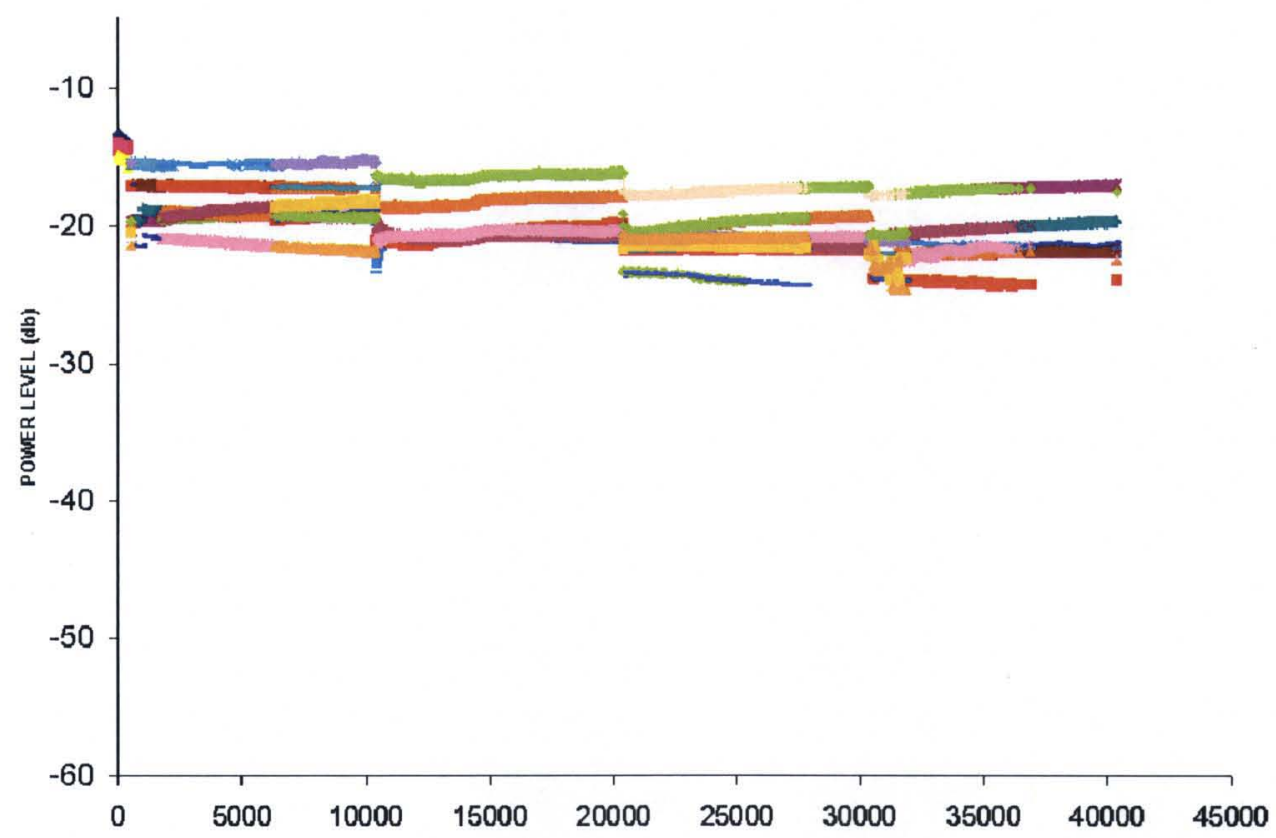

Figure 5 - Power Levels Show Only Moderate Levels of Attenuation 
A second strand with a single 3-FBG fiber attached was then tested as illustrated in the figures 6 below. Fewer spurious peaks developed on this test and less sorting of the data was required. Again, the extensometer strain is higher than those from the FBGs. The extensometer had a nominal gage length of 4.4 inches.

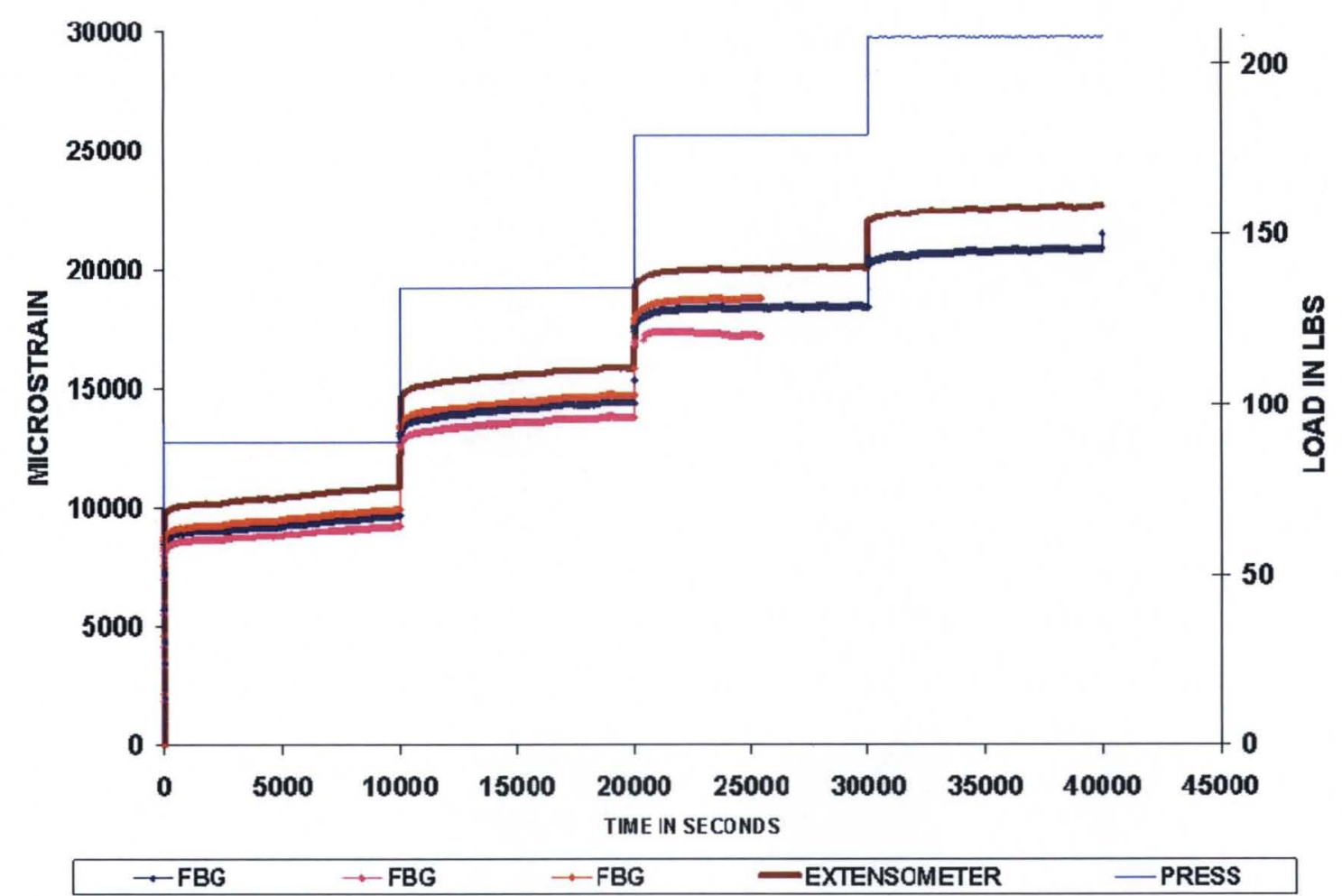

Figure 6 - Load and Microstrain Data for Extensometer and FBGs

\section{Discussions}

Although we had many problems with the sm125 interrogation system, quantitative observation can be made. The strain in a homogenous system under a constant load is the same throughout. However, in the case of a 4650 denier Kevlar strand this may not be the case, since the strand or tow is made of smaller bundles. Under strain individual bundles may have higher strain load than adjacent bundles. Since the FBG is small compared to the width of the 4650 denier strand it cannot measure the average strain over the Kevlar strand. But each FBG measures locally the strain of the tow.

For example, in figure 4 there is an 800 microstrain spread. A close inspection will reviled that many of the sensors were measuring the same strain load. It would appear that these sensors were either on the same bundle alone the length of the strand or two bundles were under identical loads.

Similarly, we see a spread over the three FBG instrumented strand in figure 6 . Here the strain difference is smaller. It was also under less twisting which led to peak splitting. Nonetheless, local strain can be measured with FBG sensors. For composite overwrapped pressure vessel localized high stress fields can potentially be an area of stress rupture. 


\section{Conclusion}

We have performed tensile creep testing 1987 DuPont manufactured 4560 denier Kevlar/Epoxy Strands. These stands are from the same stock used to manufacture composite overwrapped pressure for the Space Shuttle. FBG were used to measure the localized strain fields. A micron optics sm125 integrator was for peak wavelength detection which proved to be very difficult to extract useful data.

Quantitatively, the data showed that localized strain field can be observed. The local strain field is a strong indication of stress rupture in COPV.

\section{References}

1. J. Grant, C. Banks, "Strain Measurements Using FBGs on Composite Overwrapped Pressure Vessels (COPV) in Stress Rupture Test", Proc. of SPIE sensor System and Networks: Phenomena, Technology, and Applications for NDE and Health Monitoring, San Deigo, CA, vol. 6530, pp. 653011-1-653011-10, 2007.

2. J. Derieck, W. De Waele, P. Verleysen, "Monitering of fibre reinforced composites with embedded optical fibre Bragg sensors, with application to filament wound pressure vessels" NDT\&E International 34 (2001). pp 289-296.

3. G. Kister, D. Winter, R.A. Badcock, Y.M. Gebremichael, W.J.O. Byle, B.T. Meggitt, K.T.V. Grattan, G.F. Fernando, " Structure health monitoring of a composite bridge using Bragg grating sensors. Part 1: Evaluation of Adhesives and protection systems for the optical sensors" Engineering Structuring 29 (2007) 440 -448. 